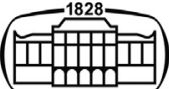

AKADÉMIAI KIADÓ

Interventional Medicine and Applied Science

11 (2019) 4, 221-223

DOI:

10.1556/1646.2020.00005

(c) 2019 The Author(s)

\title{
Pulsation of catheter during coronary angiography: Is it a sign of severe aortic regurgitation?
}

\section{MUZAFFER KAHYAOGLU ${ }^{1 *} \oplus$, CETIN GECMEN ${ }^{2}$ and OZKAN CANDAN $^{2}$}

\author{
${ }^{1}$ Department of Cardiology, Health Science University, Umraniye Training and Research Hospital, \\ Istanbul, Turkey \\ ${ }^{2}$ Department of Cardiology, Kartal Kosuyolu Heart \& Research Hospital, Istanbul, Turkey
}

Received: April 25, 2019 - Accepted: August 26, 2019

Published online: July 17, 2021

\section{RESEARCH ARTICLE}

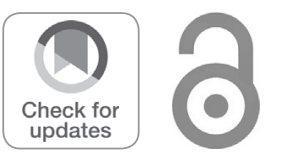

* Corresponding author. Department of Cardiology, Health Science University, Umraniye Training and Research Hospital, 34764, Umraniye, Istanbul, Turkey. Tel.: +90 5062333599; fax: +90 00216327121.

E-mail: mkahyaoglu09@hotmail.com

\section{ABSTRACT}

A 48-year-old male patient was admitted to our outpatient clinic with complaints of shortness of breath. He also had a holo-diastolic murmur at the right sternal border and an apical impulse being displaced laterally and inferiorly. Transthoracic echocardiography showed a severe aortic regurgitation without aortic valve stenosis and a mildly dilated left ventricle accompanied by an ejection fraction of $55 \%$. The aortic regurgitation jet was eccentric and there were significant holodiastolic flow reversals in the descending thoracic aorta. Surgical management was advised for this patient because of symptomatic severe aortic regurgitation. Then, the patient underwent preoperative coronary angiography through the right femoral artery route. The left coronary ostium could be engaged with a 6 Fr Judkins left diagnostic catheter; however, the catheter jumped through the ascending aorta. Afterwards, the catheter was engaged and again jumped through the ascending aorta. Engagement and jumping cycles observed between successive systole to diastole. In our opinion, this catheter movement is explained by wide pulse pressure, like the severe characteristic physical findings of severe aortic regurgitation. Further studies are needed to understand whether this catheter movement is angiographically evidence of severe aortic regurgitation.

\section{KEYWORDS}

aortic regurgitation, coronary angiography, sign

\section{BACKGROUND}

Aortic regurgitation (AR) is a valvular pathology that is caused by inadequate closure of the aortic valve leaflets. AR is usually asymptomatic and suspected by physical examination and generally diagnosed and evaluated by echocardiography. Herein, we report pulsation of catheter during preoperative angiography of a patient who underwent surgical aortic valve replacement due to severe aortic regurgitation.

\section{CASE REPORT}

A 48-year-old male patient was admitted to our outpatient clinic with complaints of shortness of breath. His symptoms worsened over the last 6 months and the degree of dyspnea at admission corresponded to New York Heart Association grade II-III. His medical history was unremarkable for any chronic illness. Upon physical examination, his blood pressure was measured as $145 / 50 \mathrm{mmHg}$, heart rate as $94 \mathrm{bpm}$, and oxygen saturation as $96 \%$. $\mathrm{He}$ also had a holo-diastolic murmur at the right sternal border and an apical impulse being displaced laterally and inferiorly. The electrocardiogram showed non-specific T-wave 
changes. Transthoracic echocardiography showed a severe aortic regurgitation without aortic valve stenosis and a mildly dilated left ventricle accompanied by an ejection fraction of 55\% (Fig. 1a and b). The aortic regurgitation jet was eccentric and there were significant holodiastolic flow reversals in the descending thoracic aorta (Fig. 1c). The aortic valve was slightly thickened and calcified and could not be clearly demonstrated as either bicuspid or tricuspid. The ascending aorta was measured as $3.9 \mathrm{~cm}$. Surgical management was advised for this patient because of symptomatic severe aortic regurgitation. Then, the patient underwent preoperative coronary angiography through the right femoral artery route which showed $50 \%$ stenosis of the left anterior descending artery. Also, the left coronary ostium could be engaged with a 6 Fr Judkins left diagnostic catheter; however, the catheter jumped through the ascending aorta. Afterwards, the catheter was engaged and again jumped through the ascending aorta. Engagement and jumping cycles observed between successive systole to diastole (Fig. 2a-c and Video 1, 2). Non-critic lesions were seen in the circumflex and right coronary artery and the patient underwent surgical aortic valve replacement.

\section{DISCUSSION}

Aortic regurgitation is a valvular pathology that is caused by inadequate closure of the aortic valve leaflets. Aortic regurgitation can be caused by primary disease of the aortic valve leaflets and/or abnormalities of the aortic root and ascending aortic geometry [1]. The inability of the aortic valve leaflets to remain closed or coapted during diastole results in a portion of the left ventricular stroke volume leaking back from the aorta into the left ventricle. The added volume of regurgitant blood produces an increase in the left ventricular end-diastolic volume and an elevation in wall stress. This situation results in ventricular hypertrophy and dilatation $[2,3]$. The combination of left ventricular eccentric hypertrophy and chamber
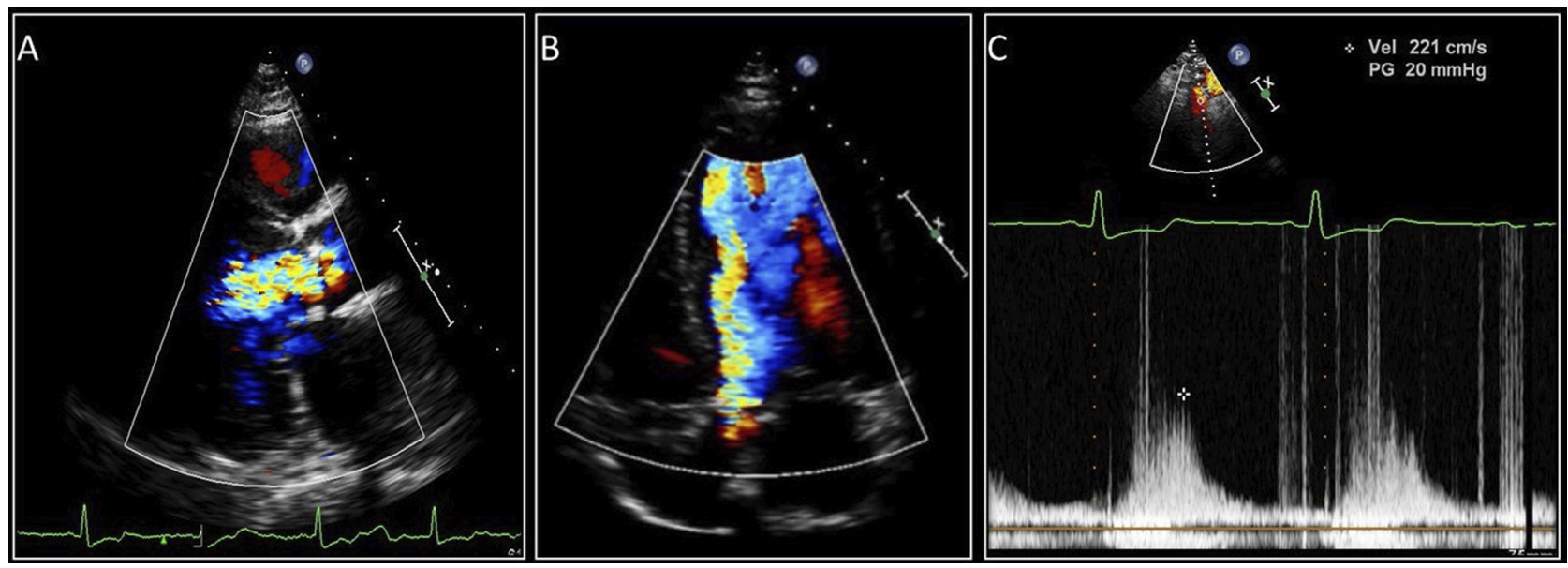

Fig. 1. a. Transthoracic echocardiography parasternal long-axis view shows severe aortic regurgitation. b. Transthoracic echocardiography apical 5-chamber view shows severe aortic regurgitation. c. Transthoracic echocardiography suprasternal view shows holodiastolic flow reversals in the descending thoracic aorta
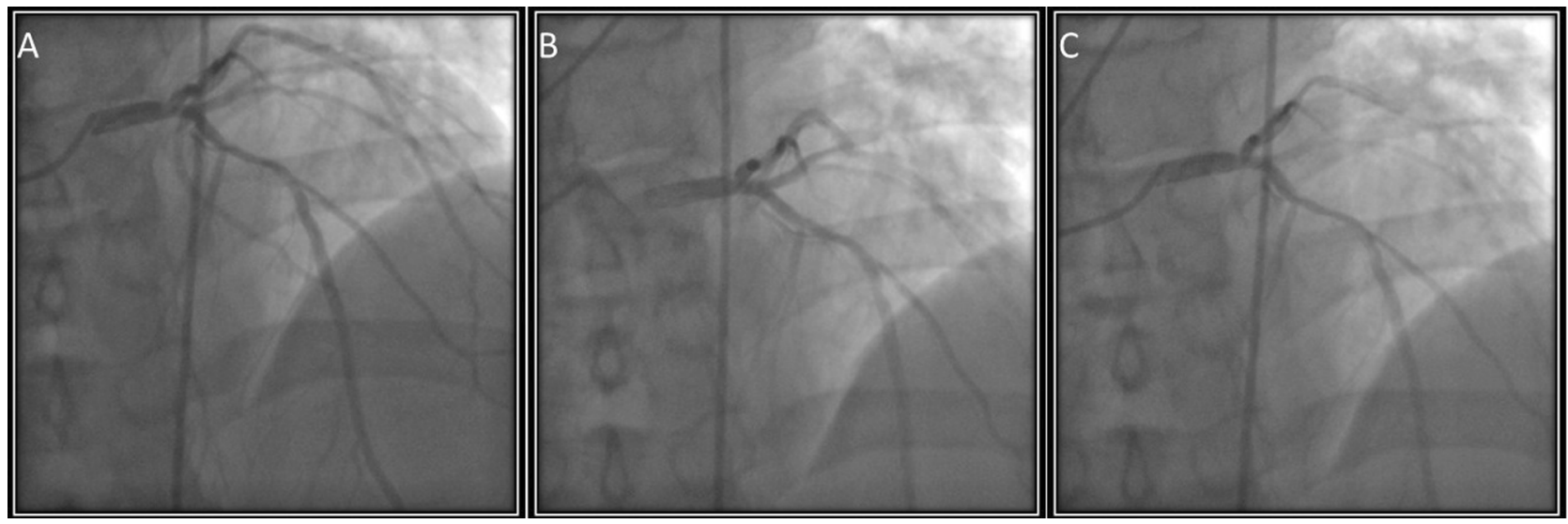

Fig. 2. a. Left coronary ostium was engaged with a left diagnostic catheter in the right cranial oblique view. b. The catheter jumped through the ascending aorta in the right cranial oblique view. c. Left coronary ostium was engaged again with the catheter in the right cranial oblique view 
enlargement raises the total stroke volume. The increased stroke volume results in abrupt distension of the peripheral arteries and an elevation in systolic pressure. Regurgitation back into the left ventricle then leads to a rapid fall in pressure with quick collapse of the arteries and a low diastolic pressure that may approach zero in severe disease. The resulting wide pulse pressure leads to a number of characteristic physical findings such as de Musset's sign, Duroziez sign, etc $[4,5]$.

$\mathrm{AR}$ is usually suspected by physical examination and generally diagnosed and evaluated by echocardiography (Figures 1 and 2). Cardiac catheterization is not routinely recommended and it is indicated for diagnosis and evaluation when noninvasive tests are equivocal or for hemodynamic assessment of AR or preoperative coronary angiography [6]. Myocardial ischemia and angina pectoris can occur even in the absence of coronary obstruction in patients with severe aortic regurgitation [7].

Myocardial ischemia may cause oxygen supply/demand mismatch because of ventricular hypertrophy or dilatation, as well as retrograde coronary artery flow $[8,9]$. Retrograde coronary flow and diastolic narrowing of the epicardial coronary artery have been reported in severe aortic regurgitation [10].

\section{CONCLUSION}

As to our knowledge, during coronary angiography, catheter movement as in our case has not been reported. In our case, we observed that 6 Fr Judkins left diagnostic catheter was engaged to the left coronary ostium during diastole, then the catheter was jumped through the ascending aorta during systole. In our opinion, this catheter movement is explained by wide pulse pressure, like the severe characteristic physical findings of severe aortic regurgitation. Presence of this finding during cardiac catheterization and coronary angiography may be a marker for aortic regurgitation. In the presence of this finding, performing aortography or evaluation of aortic valve by echocardiography may be required. We think that further studies should be conducted whether this catheter movement is an angiographic sign of severe aortic regurgitation and to determine its relationship between specific patient groups such as patients with aortic pathologies and aortic valve diseases.

Funding sources: Nothing declared.

Authors' contribution: All authors agreed to be accountable for all aspects of the work and ensuring accuracy and integrity and approved the final version of this manuscript.
Conflict of interest: The authors have no potential conflict of interest to disclose.

\section{ACKNOWLEDGMENTS}

None.

\section{SUPPLEMENTARY MATERIAL}

Supplementary data to this article can be found online at https://doi.org/10.1556/1646.2020.00005.

\section{REFERENCES}

[1] Iung B, Baron G, Butchart EG, Delahaye F, Gohlke-Bärwolf C, Levang OW, et al. A prospective survey of patients with valvular heart disease in Europe: the Euro heart survey on valvular heart disease. Eur Heart J 2003;24:1231-43.

[2] Ross J, Jr. Adaptations of the left ventricle to chronic volume overload. Circ Res 1974;35:64-70.

[3] Tezuka F. Morphometric analysis of cardiac hypertrophy: left ventricular shape and number of muscle-fiber layers across left ventricular wall. Tohoku J Exp Med 1982;138:1-6.

[4] Sapira JD. Quincke, de Musset, Duroziez, and Hill: Some aortic regurgitations. South Med J 1981;74:459-67.

[5] Hegglin R, Scheu H, Rothlin M. Aortic insufficiency. Circulation 1968;38:77-92.

[6] Nishimura RA, Otto CM, Bonow RO, Carabello BA, Erwin JP 3rd, Guyton RA, et al. American College of Cardiology/ American Heart Association Task Force on Practice Guidelines. 2014 AHA/ACC guideline for the management of patients with valvular heart disease: a report of the American College of Cardiology/American Heart Association Task Force on Practice Guidelines. J Am Coll Cardiol 2014;63:57-185.

[7] Segal J, Harvey WP, Hufnagel C. A clinical study of one hundred cases of severe aortic insufficiency. Am J Med 1956;21:200-10.

[8] Carroll RJ, Falsetti HL. Retrograde coronary artery flow in aortic valve disease. Circulation 1976;54:494-9.

[9] Aksoy S, Cam N, Guney MR, Gurkan U, Oz D, Poyraz E, et al. Myocardial ischemia in severe aortic regurgitation despite angiographically normal coronary arteries. Tohoku J Exp Med 2012; 226:69-73

[10] Tsuiki K, Watanabe M, Ikeda H, Ohta I, Yamaguchi S, Kobayashi $\mathrm{T}$, et al. Segmental diastolic narrowing of epicardial coronary arteries in aortic regurgitation. Phase analysis by quantitative angiography of coronary artery diameter change during cardiac cycles. Heart Vessels 1990;6:48-54. 